\title{
Impacto das Tecnologias de Informação e Comunicação na Técnica Psicoterápica Psicanalítica
}

\author{
Luan Paris Feijó ${ }^{*}, 1$ \\ Orcid.org/0000-0002-7587-3987 \\ Nathália Bohn Silva ${ }^{1}$ \\ Orcid.org/0000-0002-0384-7579 \\ Silvia Pereira da Cruz Benetti ${ }^{1}$ \\ Orcid.org/0000-0001-8557-2216
}

${ }^{1}$ Universidade do Vale do Rio dos Sinos, São Leopoldo, RS, Brasil

\section{Resumo}

As Tecnologias de Informação e Comunicação (TICs) têm ingressado em diversos contextos, entre eles, os consultórios que oferecem serviço psicoterápico presencial. Nessa perspectiva, o uso desses recursos impacta em conceitos técnicos da psicoterapia psicanalítica. Portanto, o objetivo deste estudo foi o de compreender como os psicoterapeutas de abordagem psicodinâmica percebem os impactos na técnica psicoterápica psicanalítica presencial. Foi realizada uma pesquisa qualitativa e exploratória com 11 psicólogos que tinham especialização em psicoterapia de orientação psicanalítica. Eles responderam à entrevista semiestruturada e a uma ficha de dados sociodemográficos. Os dados foram analisados por meio de análise temática. Os resultados evidenciaram impactos relacionados à aliança terapêutica, à neutralidade, ao setting psicoterápico e à transferência, indicando que a utilização pode proporcionar diferentes compreensões da relação terapêutica e da dinâmica de funcionamento psíquico do paciente. O estudo contribuiu para auxiliar os profissionais no manejo das TICs em psicoterapia e para fomentar a reflexão sobre a melhor forma de conjugar a tecnologia com a prática clínica presencial. Torna-se importante investigar as vivências dos psicoterapeutas e a sua formação acadêmica para ampliação das discussões sobre a temática.

Palavras-chave: Psicologia Clínica, Psicoterapia Psicodinâmica, mídias sociais e redes sociais.

\section{Impact of Information and Communication Technologies on the Psychoanalytic Psychotherapeutic Technique}

\begin{abstract}
Information and Communication Technologies (ICTs) have penetrated various contexts, including clinics that provide face-to-face psychotherapy services. From this perspective, the use of these resources impacts on technical concepts of psychoanalytic psychotherapy. Therefore, the aim of this study was

* Endereço para correspondência: Universidade do Vale do Rio dos Sinos, Av. Unisinos, 950, Cristo Rei, São Leopoldo, RS, Brasil 93022-750. Fone: (51) 99180-1619. E-mail: lparisf@gmail.com Apoio financeiro: À Coordenação de Aperfeiçoamento de Pessoal de Nível Superior pela concessão do auxílio financeiro, em forma de bolsa de mestrado, para viabilização desta pesquisa.
\end{abstract}


to understand how psychodynamic psychotherapists perceive the impacts of ICTs on the face-to-face psychoanalytic technique. A qualitative and exploratory study was carried out with 11 psychologists, specialized in psychoanalytic psychotherapy. They responded to a semi-structured interview and completed a sociodemographic data sheet. The data were analyzed through thematic analysis. The results evidenced impacts related to the therapeutic alliance, neutrality, psychotherapeutic setting, and transference, indicating that the use may provide different comprehensions of the therapeutic relationship and the psychic functioning of the patient. The study contributes as an aid for professionals to manage ICTs in psychotherapy and encourages reflection on how best to combine technology with face-to-face clinical practice. It is important to investigate the experiences of psychotherapists and their academic training to broaden the discussions on the subject.

Keywords: Clinical Psychology, Psychodynamic Psychotherapy, social media and social networks.

\section{Impacto de las Tecnologías de Información y Comunicación en la Técnica Psicoterápica Psicoanalítica}

\section{Resumen}

Las Tecnologías de Información y Comunicación (TICs) han entrado en diversos contextos, entre ellos, los consultorios que ofrecen servicio psicoterápico presencial. En esa perspectiva, el uso de esos recursos impacta en conceptos técnicos de la psicoterapia psicoanalítica. Por lo tanto, el objetivo de este estudio fue el de comprender cómo los psicoterapeutas de abordaje psicodinámico perciben los impactos en la técnica psicoterápica psicoanalítica presencial. Se realizó una investigación cualitativa y exploratoria con 11 psicólogos que tenían especialización en psicoterapia de orientación psicoanalítica. Respondieron a la entrevista semiestructurada ya una ficha de datos sociodemográficos. Los datos se analizaron mediante análisis temáticos. Los resultados evidenciaron impactos relacionados a la alianza terapéutica, a la neutralidad, al ajuste psicoterápico ya la transferencia, indicando que la utilización puede proporcionar diferentes comprensiones de la relación terapéutica y de la dinámica de funcionamiento psíquico del paciente. El estudio contribuyó a auxiliar a los profesionales en el manejo de las TICs en psicoterapia y para fomentar la reflexión sobre la mejor forma de conjugar la tecnología con la práctica clínica presencial. Es importante investigar las vivencias de los psicoterapeutas y su formación académica para ampliar las discusiones sobre la temática.

Palabras clave: Psicología Clínica, Psicoterapia Psicodinámica, medios de comunicación sociales, red social.

Estima-se que 85,6 milhões de brasileiros, $49,4 \%$ da população, utilizem a Internet por meio de microcomputador, telefone móvel, $t a-$ blet e outros recursos tecnológicos. Com base nisso, apresenta-se um panorama em que os recursos tecnológicos de informação e de comunicação (TICs) estão cada vez mais presentes na sociedade brasileira (Instituto Brasileiro de Geografia e Estatística [IBGE], 2013).

Por meio da Internet, são acessados WhatsApp, Messenger, Telegram, Facebook e outras formas tecnológicas de comunicação e de informação. Essas ferramentas proporcionam a troca de comunicações virtuais entre duas ou mais pessoas, objetivando produzir, manipular e compartilhar informações (Barcellos et al., 2015; Distéfano, Mongelo, O'Conor, \& Lamas, 2015).

Além da utilização na vida diária, essas formas de comunicação podem surgir também no contexto clínico de psicoterapia (Zilberstein, 2015). Psicoterapia é a prática profissional realizada por psicólogos, que utilizam métodos sistemáticos e técnicas cientificamente reconhecidas para analisar e compreender indivíduos ou gru- 
pos, de forma sigilosa e ética (Conselho Federal de Psicologia [CFP], 2012).

Por estarem presentes também no contexto psicoterápico, Gabbard, Kassaw e Perez-Garcia (2011) sugerem que profissionais da área da saúde possam dispor de mecanismos que preservem sua identidade profissional, a fim de não comprometer o trabalho psicoterápico. Ademais, preconizam que as instituições de formação possam ensinar os alunos sobre as fronteiras na relação terapeuta e paciente e as questões éticas relacionadas à tecnologia. No entanto, as recomendações enfocam a proteção do terapeuta, possibilitando espaço para discussão no que concerne aos impactos dos recursos tecnológicos na técnica e no processo psicoterápico, especialmente na abordagem psicodinâmica. Em geral, os psicoterapeutas dessa teoria adotam intervenções menos focais, mais interpretativas em uma relação presencial (Hallberg \& Lisboa, 2016).

A literatura nacional e internacional apresenta uma série de artigos relacionando psicoterapia e recursos tecnológicos (TICs). Grande parte desses estudos estão voltados para psicoterapia a distância (Pieta \& Gomes, 2014; Pieta, Siegmund, Gomes, \& Gauer, 2015). A maioria das pesquisas tem origem nos Estados Unidos, onde a prática de terapias online é reconhecida e aceita (Crestana, 2015). Além disso, os estudos relacionando TICs com a psicoterapia também versam sobre treinamento e supervisão a distância, mas poucos sobre a técnica no contexto presencial (Barnett, 2011).

Os artigos que discutem sobre as TICs como recursos tecnológicos na psicoterapia presencial, principalmente na América do Sul, são escassos ou não estão indexados nas bases de dados digitais (Pires, 2015). Entretanto, diversos autores sugerem estudos sobre o uso e manejo dos recursos tecnológicos em psicoterapia presencial como uma possibilidade a ser mais bem compreendida para o aprimoramento da técnica psicoterápica, uma vez que há poucas evidências clínicas sobre o assunto (Barcellos et al., 2015; Hallberg \& Lisboa, 2016; Neves \& Silva, 2014; Pires, 2015).

Estudos evidenciam que o uso dos recursos tecnológicos de comunicação e informação, como o envio de mensagens de texto/WhatsApp, poderia aumentar a aderência ao tratamento e favorecer a aliança terapêutica (Bunge, López, Mandil, Gomar, \& Borgialli, 2009; Distéfano et al., 2015). A aliança terapêutica é um fator comum às abordagens psicoterápicas, composta do acordo nos objetivos e nas tarefas do tratamento, além do estabelecimento de um vínculo positivo de confiança e apego entre a dupla terapeuta e paciente que possibilita o trabalho psicoterápico (Oliveira \& Benetti, 2015). Contudo, os desafios para o incremento dessa prática - TICs na clínica, estariam relacionados aos receios e rechaços dos usuários, bem como uma possível interferência na relação com o terapeuta (Miró, 2007).

Embora as pesquisas de Bunge et al. (2009) e de Distéfano et al. (2015) discutam os recursos tecnológicos na psicoterapia de forma mais abrangente, o estudo de Barcellos et al. (2015) especifica o uso para a técnica psicoterapêutica psicanalítica. Para as autoras, as novas formas de comunicação acabam por serem situações extra-setting que, em muitos casos, não estão incluídas nas regras e no contrato estabelecido entre a dupla. A comunicação através dos recursos tecnológicos poderia expressar tanto as ações comunicativas, como também os actings e as resistências. Assim, essas situações precisariam ser trabalhadas na relação presencial com o paciente (Barcellos et al., 2015; Sfoggia et al., 2014).

Sob esse prisma, a psicoterapia psicodinâmica centra-se nas recomendações legadas por Freud que se fundamentam na livre associação de conteúdos ocorrida na mente do paciente, na neutralidade e no anonimato do psicoterapeuta. A tarefa do terapeuta seria de tornar consciente o inconsciente através das intervenções psicanalíticas (Gabbard, 2015). Com elas, os conflitos do analisando/paciente poderiam ser ressignificados e passíveis de serem elaborados. Para isso, a dupla estabeleceria regras que norteariam o trabalho analítico e que favoreceriam o estabelecimento de uma neurose de transferência no setting terapêutico (Freud, 1912/1996a; Zimerman, 1999). No entanto, quando se trata da inserção das mídias sociais no contexto psicoterápico, a literatura psicanalítica discute o uso de forma cautelosa, uma vez que esses dispositivos aca- 
bam por influenciar no que tange a noção das regras técnicas, de setting (Crestana, 2015; Luz, 2015; Stadter, 2013), neutralidade (Kowacs, 2014; Sfoggia et al., 2014), transferência (Barbosa et al., 2013; Kowacs, 2014) e aliança terapêutica (Distéfano et al., 2015).

Embora o tema seja discutido há algum tempo, parece não haver consenso entre os profissionais atuantes na abordagem psicoterápica psicanalítica sobre como utilizá-las e manejá-las na relação presencial (Barcellos et al., 2015; Crestana, 2015). Ainda existe uma carência de pesquisas envolvendo essa abordagem, se comparadas a outras abordagens como a Terapia Cognitiva Comportamental (Hallberg, 2015; Machado et al., 2016); além disso, pouco se discute sobre a utilização do material clínico, as possibilidades e os limites dos recursos tecnológicos em psicanálise (Barcellos et al., 2015; Crestana, 2015; Machado et al., 2016). Portanto, este artigo tem como objetivo compreender como os psicoterapeutas de abordagem psicodinâmica percebem os impactos na técnica psicoterápica psicanalítica presencial em relação à utilização dos recursos tecnológicos de informação e comunicação com os seus pacientes.

\section{Método}

\section{Delineamento}

O presente estudo está pautado em uma abordagem qualitativa (Breakwell, Hammond, Five-Schaw, \& Smith, 2010) e exploratória (Gerhardt \& Silveira, 2009). Para qualificar o procedimento metodológico da presente pesquisa, foi utilizado o ChecklistConsolidated criteria for reporting qualitative research (COREQ) com o intuito de apresentar de forma detalhada o relato dos dados (Tong, Sainsbury, \& Craig, 2007). Os critérios do instrumento estão dispostos em 32 itens e divididos em três domínios, o primeiro refere-se à equipe de pesquisa, o segundo ao desenho do estudo e o terceiro à análise $\mathrm{e}$ às conclusões (Tong et al., 2007).

\section{Participantes}

Participaram desta pesquisa 11 psicólogos, sendo dez mulheres e um homem, com forma- ção em nível de pós-graduação em psicoterapia de orientação psicanalítica. Cabe destacar que os participantes selecionados concluíram a formação em psicoterapia psicodinâmica em diferentes centros de formação da região metropolitana de Porto Alegre no Rio Grande do Sul. Esse procedimento foi adotado com o intuito de selecionar uma amostra que embora estudasse a mesma abordagem teórica, havia discutido os conceitos psicanalíticos sob perspectivas diferentes e realizado supervisão com professores distintos. Além disso, foi utilizado o critério de saturação dos dados (Fontanella, Ricas, \& Turato, 2008) para o número final de participantes, assim, quando se percebeu que as informações emitidas pelos participantes passaram a não apresentar novos conteúdos, encerraram-se as entrevistas.

\section{Instrumentos}

Os instrumentos utilizados para a coleta de dados foram: (1) ficha de dados sociodemográficos e (2) entrevista semiestruturada com questões norteadoras. A entrevista semiestrutura foi desenvolvida a partir de um estudo-piloto realizado com seis psicólogos que possuíam formação em nível de pós-graduação em psicoterapia de orientação psicanalítica. Esses profissionais responderam a uma primeira versão do questionário com 19 perguntas abertas. Após análise das respostas, organizou-se uma nova versão do questionário com nove questões abertas distribuídas em quatro categorias e acrescidas de tópicos exploratórios. Os temas e as perguntas exploratórias estavam centrados no ingresso dos recursos tecnológicos na prática clínica, nos impactos desses recursos na técnica psicanalítica, na formação do profissional e no manejo de casos clínicos através dos recursos tecnológicos.

\section{Procedimentos de Coleta dos Dados}

Os participantes do estudo foram contatados por meio de ligação ou e-mail. Após o aceite de participação, foi agendado um dia e horário com o profissional para realização da entrevista. Os participantes assinaram o Termo de Consentimento Livre e Esclarecido em duas vias. Após esse procedimento, deu-se início à gravação do áudio da entrevista. Primeiro, foi preenchida a fi- 
cha de dados sociodemográficos e posteriormente a entrevista semiestruturada. As entrevistas tiveram duração média de 35 minutos.

\section{Procedimentos Éticos}

A pesquisa esteve amparada pela Resolução 510/2016 (2016) do Conselho Nacional de Saúde, órgão do Ministério da Saúde e foi aprovada pelo Comitê de Ética da Universidade do Vale do Rio dos Sinos - Unisinos (CAE: $60157816.8 .0000 .5344)$ por meio do parecer $n^{\circ}$ 1.790 .149 .

\section{Procedimentos de Análise dos Dados}

Os dados foram analisados através de análise temática realista, que é entendida como uma análise dos temas predominantes nas entrevistas e que opera com a realidade dos participantes (Braun \& Clarke, 2006).

Inicialmente, foi transcrito o áudio das entrevistas. Após a transcrição, o material foi lido de forma livre para se obter uma maior aproximação com os dados coletados. Em uma segunda leitura, foram assinaladas as unidades temáticas emergentes. Na terceira leitura, as unidades temáticas foram agrupadas e geraram os temas da pesquisa. Por fim, as comunicações assinaladas foram divididas e organizadas em temáticas. As entrevistas foram analisadas e categorizadas por dois juízes independentes. Nos casos em que houve divergência, uma terceira juíza, foi convidada a categorizar os materiais. Portanto, para a solução dos casos conflitantes foi adotado o consenso entre juízes.

As comunicações foram reunidas em temas, sendo estes: (1) experiência dos profissionais na utilização das tecnologias de informação e comunicação na clínica psicoterápica psicodinâmica; (2) impactos das tecnologias de informação e comunicação na técnica psicanalítica e (3) formação do profissional para manejo dos recursos tecnológicos na clínica.

Neste artigo, serão apresentados os resultados do tema (2) impactos das tecnologias de informação e comunicação na técnica psicanalítica, o qual foi dividido nas seguintes categorias: (a) aliança terapêutica; (b) neutralidade; (c) enquadre e (d) transferência. Para elucidar sobre os impactos na técnica, serão apresentadas as vinhetas. Os demais temas, (1) experiência dos profissionais na utilização das tecnologias de informação e comunicação na clínica psicoterápica psicodinâmica e (3) a formação do profissional para manejo dos recursos tecnológicos na clínica, foi objeto de discussão em outro artigo elaborado pelos autores (Feijó, Silva e Benetti, 2018).

\section{Resultados}

Os terapeutas possuíam experiência clínica de 10,18 anos ( $d p=5,30$ anos), concluíram a graduação há 10,55 anos $(d p=5,20$ anos $)$ e a especialização há 6,46 anos ( $d p=3,36$ anos). Tinham idades entre 27 e 55 anos $(\mathrm{m}=34,7$ anos e $d p=7,37$ anos). Além de todos terem a titulação de especialista em psicoterapia de orientação psicanalítica, três das participantes realizaram o mestrado na área de psicologia clínica, em grupos de pesquisa com enfoque na abordagem psicanalítica. Destas três participantes, duas eram docentes de instituições de formação em psicoterapia psicanalítica no Rio Grande do Sul.

No que se refere à utilização dos recursos tecnológicos de informação e comunicação, todos os participantes utilizavam o WhatsApp em sua prática clínica. Três psicoterapeutas foram contatados pelo Messenger e nove por meio do Facebook. Nenhum deles foi procurado no Instagram ou em outras formas de tecnologias de informação e comunicação.

A partir da análise dos dados, os relatos foram agrupados em unidades temas e posteriormente geraram as categorias de análise temática. Esses aspectos foram identificados nas seguintes categorias: (a) aliança terapêutica; (b) neutralidade; (c) Setting terapêutico e (d) transferência. Assim, as unidades temáticas representam o agrupamento das entrevistas e relacionam os impactos na técnica psicoterápica psicanalítica.

\section{Aliança Terapêutica}

A primeira categoria, (a) aliança terapêutica, apresentou resultados temáticos relacionados à adesão ao tratamento e a identificação das fragilidades psíquicas do paciente, conforme a Tabela 1 . 
Tabela 1

Impactos na Aliança Terapêutica

\begin{tabular}{ll}
\hline Impacto & \multicolumn{1}{c}{ Vinheta } \\
\hline Adesão ao tratamento & $\begin{array}{l}\text { "Acho que quando tu te mostras mais disponível para alguns pacientes, eles } \\
\text { tendem a se vincular mais rápido" (Participante 3). }\end{array}$ \\
$\begin{array}{l}\text { Identificação } \\
\text { das fragilidades psíquicas } \\
\text { do paciente }\end{array}$ & $\begin{array}{l}\text { eu vi que tu olhou o WhatsApp e tu não me respondeu . . . [terapeuta analisa] } \\
\text { Desse modo, a fragilidade dos pacientes vai aparecer, muitas vezes nesse } \\
\text { momento. (Participante 5) }\end{array}$ \\
\hline
\end{tabular}

Nesta categoria, os participantes relataram que os recursos tecnológicos impactam de diferentes formas na aliança terapêutica. Percebeu-se como impactos positivos na utilização das TICs que os pacientes se sentem acolhidos, aderem melhor ao tratamento e sentem seus psicoterapeutas mais empáticos, pois estavam disponíveis nas mídias sociais.

Ademais, as tecnologias auxiliavam os terapeutas a compreender as fragilidades psíquicas do paciente, por exemplo, se o cliente precisava que o terapeuta o gratificasse respondendo às mensagens ou que o frustrasse visualizando e deixando a situação para ser trabalhada em sessão. Nesse sentido, compreendeu-se que, se o te-

\section{Tabela 2}

Impactos na Neutralidade rapeuta intervém de forma mais ativa ou não, por meio das tecnologias de informação e comunicação, ele poderia impactar na aliança terapêutica, e isso influenciaria na decisão do paciente de manter ou não o tratamento. Portanto, o manejo profissional variava conforme as necessidades que o terapeuta julgava serem importantes para o tratamento.

\section{Neutralidade}

A segunda categoria (b) neutralidade apresentou resultados temáticos centrados na preocupação com o posicionamento dos psicoterapeutas participantes frente às TICs; a exposição pessoal e a percepção da neutralidade na atualidade. A Tabela 2 sintetiza os resultados encontrados.

Impacto

Vinheta

... sempre penso no WhatsApp, porque o Facebook é uma preocupação que eu tinha de ficar [exposto], a gente coloca ideias, eu procuro colocar o mínimo possivel de ideias, quanto às

Posicionamento aderido por psicoterapeutas

Exposição do terapeuta

Percepção da neutralidade na atualidade minhas ideologias ou algo, até porque eu utilizo mais como uma reflexão. (Participante 2) A primeira [preocupação] delas . . como que tu vai escrever uma mensagem? Eu tomo muito cuidado quando eu vou escrever mensagem de texto para não usar gíria, para não usar abreviatura. Por exemplo, quando escrevo "o que", não coloco só "oq", escrevo o português bem correto. (Participante 10)

WhatsApp eu nunca tive uma foto minha, é uma árvore, porque dai como eu sei que o paciente acessa, a ideia de o paciente ver uma foto minha feliz, ou mais bonita, ou com filhinho, ou com cachorrinho ... momentos, ah a paciente tá sozinha e vê a fotinho de um casal feliz ali sabe? Acho que deve ser dificil. (Participante 8)

"... Poder ver a hora que o terapeuta entrou, a foto que ele usou no perfil" (Participante 6). . . . aconteceu uma situação com um adolescente. Ele encontrou meu perfil [Facebook] $e$ dava pra ver as páginas que eu tinha curtido, isso eu não sabia que dava pra fechar até então, e ele me questionou [em sessão] por que que tinha curtido tal página. (Participante 1)

. . a apesar de que isso [neutralidade] nos dias atuais é um tanto quanto ilusório porque nada mais é completamente neutro, as pessoas têm acesso à nossa vida de diferentes formas, através daquilo que a gente publica, através da nossa formação [profissional]. (Participante 7) 
Em relação à temática neutralidade, os participantes consideraram que o posicionamento dos psicoterapeutas nos recursos tecnológicos poderia impactar negativamente no tratamento, pois no WhatsApp tem exposto o horário em que visualizou suas conversas. No Facebook, quais assuntos comentou ou compartilhou, além de ter as suas imagens disponibilizadas na rede, caso não tenha configurado a privacidade. Outra situação que suscitou questionamentos, esteve relacionada à forma com que o profissional se apresenta nesses canais comunicativos, por exemplo, o terapeuta escreve uma mensagem ou publica posicionamentos nas redes sociais. Essas ocorrências podem evidenciar informações da vida privada do psicoterapeuta para além do seu exercício profissional.

Ainda sobre a neutralidade, a participante 6 relatou o exemplo em que uma paciente deprimi- da que visualizou foto da terapeuta feliz no $F a$ cebook, projetou sentimentos hostis por não ter a capacidade, pelo menos momentaneamente, de ter os mesmos sentimentos que a sua terapeuta. Por fim, os psicoterapeutas informaram nas entrevistas que com as tecnologias de informação e comunicação, as informações pessoais se tornaram mais rápidas e fáceis de serem encontradas nos diversos recursos tecnológicos.

\section{Setting Terapêutico}

Nesta categoria, os impactos percebidos pelos terapeutas estão relacionados às combinações do contrato de trabalho terapêutico, aos recursos tecnológicos como um terceiro no setting terapêutico e às situações extra-setting, entendidas como aquelas que aparecem externamente à sessão presencial. A Tabela 3 exemplifica através dos relatos das entrevistas esses impactos.

Tabela 3

Impactos no Setting Terapêutico

Impacto Vinheta

Manutenção do Contrato terapêutico

Situações

Extra-setting

Recursos tecnológicos como um terceiro no setting terapêutico
Mas muito mais naquela coisa de...ãa... marcar...des[marcar]... paciente né: Ah, ó preciso desmarcar a consulta hoje. Ah dá pra transferir? Eu preciso de uma sessão extra. [Mais] nesse sentido do que qualquer outra coisa. (Participante 4)

“. . . Aquilo começa [TICs] a ser um problema . . . quando conteúdos que deveriam ser tratados na sessão aparecem fora" (Participante 5).

Eu acho que muitas coisas podem ficar resolvidas ali no WhatsApp e não vão pra dentro do setting . . . acho que resolvendo coisas pelo WhatsApp muitas vezes a gente deixa de falar mais profundamente sobre isso na sessão. (Participante 9)

... [Eu] recebo mensagens de pacientes perversos, ou border[line], esses eu preciso ter mais cuidado, porque eles não sabem fazer uso dessas tecnologias e acabam colocando em risco o processo terapêutico ... eles acabam invadindo mais, sabendo menos o limite, né? Então, o horário não é adequado, ã, ficam te mandando mensagem né, para ver se tu realmente olhaste. (Participante 6)

Estou me questionando como é que eu manejo isso . . . principalmente na hora que eles vão contar uma situação né, por exemplo se eles pedirem pra ler a conversa do WhatsApp. Às vezes, eles tão explicando uma conversa, sei lá, com namorado e em vez de dizer: "ah, aconteceu isso, isso e isso..." eles "não, não, calma aí que eu vou ler para ti pra eu não esquecer nada". (Participante 10)
Nesta categoria, os psicoterapeutas participantes do estudo relataram que os seus pacientes tendem a solicitar sessões adicionais, remarcar e desmarcar atendimentos por meio do WhatsApp, mais do que presencialmente. A participante 4 relatou ainda que seria necessário que no próprio contrato terapêutico inicial incluísse as combinações de como poderiam ser utilizadas essas tecnologias de informação e comunicação, a fim poder estabelecer um enquadre sobre elas.

No que se referem às situações extra-setting, os relatos indicaram que os terapeutas necessi- 
tam dispensar atenção para que as comunicações que deveriam ocorrer na sessão não sejam solucionadas através do uso dos recursos tecnológicos, pois assim deixariam de ser analisadas em profundidade. Exemplos fornecidos pelos psicoterapeutas de situações extra-setting estavam relacionadas às atuações, resistências, faltas, remarcações, a conteúdos que só emergem nas TICs, mas não nas sessões e, finalmente, ao cuidado nessas situações com os pacientes limítrofes (borderline).

Já no tema recursos tecnológicos como um terceiro no setting terapêutico, os participantes apontaram que as novas ferramentas de informação e comunicação acabam por ser mais um

Tabela 4

Impactos na Transferência elemento do setting psicoterapêutico presencial, pois o paciente utilizava WhatsApp e Facebook para relatar situações ocorridas no seu dia a dia, interrompendo um relato associado livremente. Nesse mesmo tema, outra problemática iminente estava relacionada aos clientes dos participantes usarem as TICs dentro da sessão para olhar o horário, atender a ligações ou enviar mensagens.

\section{Transferência}

A categoria transferência apresentou dois temas de discussão pelos participantes, um relacionado à expressão dos desejos dos pacientes por meio dos recursos tecnológicos de informação e comunicação e outro relacionado à presença da transferência.

Impacto Vinheta

Recursos tecnológicos como representantes dos desejos dos pacientes

Manifestação da transferência nos recursos tecnológicos de informação e comunicação
Quando eu tava online à noite ou em algum momento ela [paciente] vinha querer conversar . . . foi necessário trabalhar isso na sessão várias vezes . . que a gente tinha que usufruir, vir mais, aumentar a frequência [dos atendimentos] . . . (Participante 2)

Eu fico pensando inicialmente que o trabalho da transferência, né, que é algo que é um dos pilares, como ele vai reviver situações, a gente pode, sim, usar as ferramentas [WhatsApp] pra compreender a necessidade de uma mãe muito presente, ou uma mãe muito invasiva, ou um filho de repente solicitando muito essa mãe ou esse pai dependendo da função que ele projeta . . . podendo usar disso dentro da sessão, usar esses exemplos que acontecem pra poder fomentar ideias, para interpretar... (Participante 2)
No primeiro tema, recursos tecnológicos como representantes dos desejos dos pacientes, os psicoterapeutas apresentaram relatos informando que através dessas formas de comunicação e informação seus pacientes sinalizavam suas necessidades. Nesse canal de comunicação, os participantes podiam expressar a sua angústia, podendo o terapeuta acolher ou abster-se para posteriormente discutir em sessão.

Já no segundo tema, manifestação da transferência através dos recursos tecnológicos de informação e comunicação, dez dos 11 terapeutas acreditavam que a tecnologia era um canal propício para ocorrência desta. Apenas um dos participantes acreditava que a transferência ocorreria somente na relação presencial e não através das tecnologias. $\mathrm{O}$ relato apresentado na Tabela 4 evidencia um caso em que as solicitações recorrentes ao psicoterapeuta poderiam ser compreendidas como a manifestação da transferência, e não somente ao uso indiscriminado por parte do paciente.

\section{Discussão}

A partir do relato dos participantes, há uma perceptível mudança na técnica psicanalítica com a inserção das tecnologias de informação e comunicação na prática clínica. Embora os psicoterapeutas apresentem um elevado tempo de experiência clínica e formação profissional, é possível perceber que o manejo desses recur- 
sos é cauteloso e direcionado para combinações relacionadas ao contrato psicoterapêutico de trabalho. Entretanto, as TICs têm apresentado um fértil campo para ampliação e manifestação de conteúdos transferenciais (Barbosa et al., 2013), resistências, atuações, situações extra-setting (Barcellos et al., 2015), complexidades do próprio setting terapêutico (Luz, 2015) e da aliança terapêutica (Bunge et al., 2009).

A psicoterapia psicanalítica fundamenta-se primordialmente na obra Freudiana no que se refere à associação livre de conteúdos advindos inconscientemente na mente do analisando (Freud, 1912/1996a), bem como no esforço do psicoterapeuta para que o paciente mantenha uma neurose de transferência (Freud, 1914/1996b, 1920/1996d). Partindo dessa premissa, pode-se entender que se existem conteúdos inconscientes, que não são regidos nem por regras, nem pelo tempo e podendo estes se manifestarem de diferentes maneiras, reflete-se então que uma das suas manifestações poderia ser inclusive a partir das tecnologias de informação e comunicação (Kowacs, 2014), evidenciando, assim, as necessidades, fragilidades e frustrações dos pacientes, bem como o seu funcionamento psíquico. $\mathrm{O}$ desenvolvimento do psiquismo modifica-se com as transformações culturais, e, se estamos inseridos em uma cultura tecnológica, não devemos negar a inserção das TICs na vida dos pacientes (Kowacs, 2014), pois a psicanálise precisaria se adaptar aos novos tempos, mas sem perder suas características próprias (Kowacs, 2014).

No que se refere aos resultados desta pesquisa no tema aliança terapêutica, as temáticas presentes foram adesão ao tratamento e identificação das fragilidades psíquicas do paciente. A literatura indica que os recursos tecnológicos podem favorecer a adesão à psicoterapia e a vinculação com o terapeuta (Bunge et al., 2009). Nesta pesquisa, a percepção dos profissionais participantes foi de que as TICs fortaleciam a aliança terapêutica (AT) e a adesão ao tratamento, pois favorecem o estabelecimento de uma sensação de acolhimento do terapeuta por parte do paciente. Portanto, os resultados encontrados estão em consonância com a literatura.
Além das TICs fortalecerem a AT, não foram encontrados indícios nos discursos dos participantes de que houvesse interferência na aliança terapêutica no tratamento presencial em decorrência do uso desses recursos. Em semelhança aos achados nesta pesquisa, Sucala et al. (2012) concluem em revisão sistemática de que é possível desenvolver uma aliança terapêutica eficaz utilizando a Internet, inclusive em tratamentos online, onde não há presença de uma comunicação não verbal. Nesse sentido, a importância de estabelecer um vínculo positivo no início do processo psicoterapêutico se faz importante para que o paciente se mantenha no tratamento psicológico, seja presencial ou com os recursos tecnológicos.

Referente à identificação das fragilidades psíquicas dos pacientes, percebeu-se que os terapeutas puderam usar das informações disponíveis nas TICs para complementar o processo presencial, bem como o entendimento dinâmico do seu paciente. Esse achado de pesquisa contrasta com a literatura psicanalítica, pois esta aborda a importância da relação presencial para manejo dos aspectos relacionados ao tratamento psicoterapêutico (Eizirik, Aguiar, \& Schestatsky, 2005). Por ser um tema relativamente novo com essa abordagem, diversos autores têm contribuído para diminuir a polêmica sobre o uso para concebê-las de forma positiva no processo psicoterápico (Barcellos et al., 2015; Sfoggia, et al., 2014). Os recursos tecnológicos possuem potencial para se tornar uma ferramenta aliada dos profissionais na sua prática clínica, pois auxiliam na complementação da relação entre a dupla e nas intervenções do terapeuta (Luz, 2015).

$\mathrm{Na}$ categoria neutralidade, os temas predominantes estiveram vinculados à preocupação com o posicionamento pessoal do terapeuta, à sua exposição nas TICs e à percepção da neutralidade na atualidade. Na literatura psicanalítica, Freud (1912/1996a) defendia que a abstinência e a neutralidade do psicoterapeuta eram mecanismos essenciais para se atingir os objetivos da psicoterapia. Nessa perspectiva, a preocupação dos terapeutas em manterem-se neutros e reservados fundamenta-se nas premissas da própria 
literatura psicanalítica. Entretanto, embora seja importante que os profissionais mantenham um manejo ponderado sobre o uso, restringir as tecnologias de informação e comunicação por completo talvez seja menos neutro do que utilizá-las (Barbosa et al., 2013; Luz, 2015). Portanto, Sfoggia et al. (2014) discutem que a maneira como o terapeuta e paciente usam essas tecnologias é que impactará no tratamento e não a tecnologia em si. Possivelmente, a influência negativa na neutralidade pode estar relacionada também ao fato de os terapeutas participantes vincularem o termo neutralidade à abstinência, sendo que esses conceitos possuem diferenças de entendimento.

Para Falcão (2007), a neutralidade está pautada a um analista neutro em relação às suas convicções, a seus ideais e às manifestações transferenciais. Assim, não estariam nas mãos do terapeuta impor ao paciente os seus desejos e as suas escolhas (Meissner, 1998). Já a abstinência está relacionada às gratificações substitutivas, com o intuito de que o paciente as encontre o mínimo possível ao longo do tratamento (Falcão, 2007). Contudo, o próprio Freud, em algumas situações, acabava por gratificar seus pacientes, quando os elogiava pelos relatos expressos, por exemplo (Zamfirescu, 2013). Zamfirescu (2013) discute que privar o paciente de todas as gratificações seria insuportável. Nesse sentido, pode-se pensar que usar as tecnologias com os pacientes seria uma forma, ainda que possa ser somente inicial, de acolher o paciente para que depois esse uso possa ser direcionado a outro destino, como um acréscimo na frequência das sessões ou a uma interpretação de seu uso. Ademais, parece que a tecnologia pode não afetar diretamente na neutralidade do terapeuta, mas, sim, na abstinência deste, sendo necessário o cuidado do profissional para que as TICs não sejam um caminho de gratificação substitutiva.

Em relação à percepção da neutralidade na atualidade, é notável que as tecnologias estejam cada vez mais presentes na vida pessoal e profissional dos psicoterapeutas (Hallberg \& Lisboa, 2016). Gabbard (2012) considera a perda do anonimato como uma das grandes mudanças para o trabalho psicodinâmico. Portanto, primor- dialmente, pode-se recorrer a Freud quando este escreve que algumas permissões devem ser realizadas de acordo com cada paciente e com sua individualidade, sem que se tornem excessivas (1919/1996c), e nortear-se nas recomendações de Gabbard et al. (2011) sobre a postura profissional na Internet. Esses autores recomendam que os profissionais ativem as configurações de privacidade, monitorem as informações pessoais disponíveis na Internet e não realizem buscas sobre seus pacientes na rede de dados. Essas regras podem auxiliar os profissionais a lidar com uma possível perda de uma postura neutra, ainda que esta possa não ocorrer em função do uso das tecnologias.

No que se refere ao setting terapêutico, foram encontrados resultados relacionados ao contrato terapêutico, às situações extra-setting e aos recursos tecnológicos como um terceiro no setting terapêutico. Percebeu-se nas entrevistas que o uso e manejo pelos psicoterapeutas estavam relacionados às questões específicas do contrato de trabalho e que faziam parte do enquadre, mesmo que não contratadas previamente. Barcellos et al. (2015) discutem que essas situações, embora não combinadas inicialmente pela dupla, podem ser compreendidas como parte do campo analítico, constituídas como uma construção única da dupla e que podem ser usadas para favorecer a relação terapêutica. As mesmas autoras discutem sobre essas situações serem uma expressão das ansiedades e dos conflitos dos pacientes descarregados via ato (2015).

Outro prisma para as modificações no setting por meio das TICs pode ser observado a partir do conceito de parâmetro à psicoterapia (Migone, 2013). A noção desse conceito tem como premissa explicar aquelas situações que possam transgredir o enquadre. Para a sua inclusão, o psicoterapeuta deverá estar seguro de que conseguirá dominá-lo, deverá avaliar se ele não prejudicará o setting e se terá condições de retomar às combinações originais com o seu paciente, caso seja necessário (Zimerman, 1999). Portanto, pode-se propor o uso dessas tecnologias como uma forma de concessões iniciais para que seja um canal de expressão do paciente, a fim de propô-lo como um parâmetro à técnica. Para Sfoggia 
et al., (2014), o setting externo pode tornar-se flexível, desde que o setting interno do terapeuta se mantenha preservado.

Quanto aos recursos tecnológicos como um terceiro no setting terapêutico, Stadter (2013) compreende esses novos objetos tecnológicos como um e-third (terceiro eletrônico), que ingressam nas sessões assim como um elemento de transição ingressa na vida de criança, com o intuito de que esta consiga se tornar independente das primeiras relações interpessoais. Esse terceiro eletrônico auxiliaria no estabelecimento dessas relações, embora ele também problematize que essas TICs podem se tornar recursos de dependência tecnológica (Stadter, 2013). No entanto, os recursos tecnológicos ampliam a possibilidade de que o paciente consiga expressar com maior facilidade conteúdos ainda difíceis ou intoleráveis de se apresentarem de outro modo, como presencialmente por exemplo (Migone, 2013). A função do terapeuta seria então a de captar essas ações comunicativas e poder interpretar ao seu paciente essa situação para que pudessem ser trabalhadas no contexto presencial e não somente resolvidas por meio dos recursos tecnológicos.

Por fim, os resultados da pesquisa sobre a transferência estão relacionados aos recursos tecnológicos como representantes dos desejos dos pacientes e à manifestação da transferência nos recursos tecnológicos de informação e comunicação. Com esses recursos, a transferência encontra um novo espaço para expressão (Kowacs, 2014), diminuindo o lapso de tempo referente à solidão ou exclusão e fazendo com que o paciente se permita ingressar de forma efetiva na vida do terapeuta (Kowacs, 2014), denunciando as suas necessidades afetivas. Neste estudo, a maioria dos terapeutas acredita que ocorre a manifestação da transferência por meio das tecnologias de informação e comunicação. Embora ainda não haja consenso na literatura sobre a presença ou não da transferência nas TICs (Carlino, 2011; Garcia, 2011; Kohn \& Roth, 2012; Scharff, 2013)

Para Garcia (2011), a transferência só aconteceria se houvesse a presença real da dupla na sessão. Já Kohn e Roth (2012) propõem que esta ocorre mesmo na utilização desses recursos tecnológicos. Por meio das entrevistas realizadas neste estudo, os terapeutas acreditam que há transferência por meio das TICs. Corroborando essa perspectiva, Scharff (2013) discute que os elementos transferenciais e contratransferenciais estão presentes na utilização dessas tecnologias. Para o autor, a associação livre e a transferência não são comprometidas pelos recursos tecnológicos. Ainda que as discussões dos referidos autores sejam em relação às sessões de psicoterapia online, a utilização das TICs em atendimentos psicoterápicos que possuem encontros presenciais pode ser compreendida como similar ao contato não presencial (online), pois não há presença física da dupla no uso destas tecnologias.

Contudo, a reflexão não deve se restringir à apresentação ou não de um elemento da técnica psicanalítica, mas, sim, perceber essas manifestações como um fenômeno complexo. Barbosa et al. (2013), de forma metafórica, escrevem que é necessário "jogar o bebê fora e ficar com o resto sujo da água do banho" (p. 67). A ideia implícita reside em não olhar somente a tecnologia, mas os resultados que ela traz, pois podem representar desejos, necessidades e comportamentos singulares do paciente. Portanto, acredita-se ser interessante refletir o uso das TICs, desde que não seja uma perversão tecnológica para contribuir como um espaço de comunicação transferencial do paciente. Entretanto, cabe destacar também a necessidade da ausência do terapeuta entre uma sessão e outra na vida do paciente para que ele possa subjetivar. Nesse sentido, é importante que o terapeuta possa se manter disponível e continente, mas que também possa oferecer limites e certa ausência de gratificações. Será nesse jogo transferencial que fecundará novas construções analíticas (Barbosa et al., 2013).

\section{Conclusão}

Este artigo teve como objetivo compreender como os psicoterapeutas de abordagem psicodinâmica percebem os impactos na técnica psicoterápica psicanalítica presencial em relação à utilização dos recursos tecnológicos de infor- 
mação e comunicação com os seus pacientes. O tempo de experiência clínica e de formação profissional parece não indicar diferença na percepção dos impactos na técnica psicanalítica. Os resultados evidenciam que as influências foram relacionadas à adesão ao tratamento, à identificação das fragilidades psíquicas do paciente, ao posicionamento e à exposição do terapeuta, à percepção da neutralidade, ao contrato terapêutico, às situações extra-setting, aos recursos tecnológicos como um terceiro no setting terapêutico como representantes dos desejos dos pacientes e à manifestação da transferência nos recursos tecnológicos de informação e comunicação.

Se partirmos da premissa referida na obra Freudiana de que o funcionamento psíquico dos sujeitos é regido pelo inconsciente e de que para este não há regras temporais ou espaciais, já que pode se manifestar sob diferentes formas, podemos refletir que as tecnologias de informação e comunicação servem como um canal de comunicação de conteúdos inconscientes do paciente. Cabe destacar que ainda discutimos o tema de forma cautelosa, por este ser relativamente novo na prática profissional psicodinâmica. Entretanto, como visto nos resultados deste estudo, as tecnologias de informação e comunicação podem ser auxiliares na compreensão psíquica do paciente, bem como do processo psicanalítico. Negar a tecnologia talvez fosse mais danoso ao nosso trabalho do que olhá-la com parcimônia, aceitando a sua inserção e trabalhando-a com cada paciente, afinal não há como generalizar o seu uso.

Cabe ressaltar que estamos inseridos em uma sociedade que está em constante transformação. Com isso, devemos lembrar que o estudo psicanalítico leva em consideração variáveis sociais do indivíduo, por exemplo, a transmissão de valores entre as gerações e o contexto inserido.

Acredita-se ser importante discutir a temática nos centros de formação que ensinam a psicoterapia de orientação psicanalítica para compreender quais afetos esse uso mobiliza no terapeuta, bem como instrumentalizar os profissionais para uma utilização adequada da técnica. Além disso, sempre que necessário buscar su- pervisão dos casos clínicos para aprofundamento das situações vivenciadas na clínica.

Quanto a limitações do estudo, percebe-se o uso ainda restrito das combinações contratuais por meio das TICs pelos profissionais. Possivelmente, se o manejo estivesse mais bem estabelecido entre os participantes, os resultados encontrados poderiam ser aprofundados em relação à técnica.

Em relação a direções futuras, fazem-se necessárias pesquisas quantitativas com amostras brasileiras sobre como os profissionais do nosso país percebem os impactos na técnica psicanalítica e como manejam, tanto com psicoterapeutas psicodinâmicos quanto com analistas. Ademais, é preciso analisar o uso das tecnologias de informação e comunicação em estudos de caso único delineados em pesquisas de processo e resultado em psicoterapia. Por fim, um eixo de investigação que necessita de atenção se refere a como as instituições de graduação em psicologia e pós-graduação em psicanálise têm discutido a temática com os seus alunos. A psicoterapia e as tecnologias demonstram ser temas promissores na área da psicologia, pois ampliam as possibilidades de intervenção com os indivíduos e as coletividades.

\section{Contribuições dos Autores}

Contribuição substancial no conceito e desenho do estudo: Luan Paris Feijó; Silvia Pereira da Cruz Benetti.

Contribuição na coleta de dados: Luan Paris Feijó.

Contribuição na análise e interpretação de dados: Luan Paris Feijó e Nathália Bohn da Silva.

Contribuição para a preparação do manuscrito: Luan Paris Feijó.

Contribuição à revisão crítica, agregando conteúdo intelectual: Luan Paris Feijó; Silvia Pereira da Cruz Benetti.

\section{Conflitos de interesse}

Os autores declaram não ter conflito de interesse relacionado à publicação deste manuscrito. 


\section{Referências}

Barbosa, A. M. F. D. C., Furtado, A. M., Franco, A. L. D. M., Berino, C. G. D. S., Pereira, C. R., Arreguy, M. E., \& Barros, M. J. D. (2013). As novas tecnologias de comunicação: Questões para a clínica psicanalítica. Cadernos de Psicanálise (Rio de Janeiro), 35(29), 59-75. Recuperado em http://pepsic.bvsalud.org/scielo.php?script=sci arttext\&pid=S1413-62952013000200004\&lng= $\mathrm{pt \& t} \operatorname{lng}=\mathrm{pt}$

Barcellos, E. D., Campezatto, P. V. M., Geremia, L., Vieira, M. F., Cavalheiro, R., \& Klarmann, R. P. (2015). As novas formas de comunicação e suas possíveis influências sobre o setting e o campo psicoterápico. Psicoterapia Psicanalítica, 17, $39-51$.

Barnett, J. E. (2011). Utilizing technological innovations to enhance psychotherapy supervision, training, and outcomes. Psychotherapy, 48(2), 103. doi: http://dx.doi.org/10.1037/a0023381

Braun, V., \& Clarke, V. (2006). Using thematic analysis in psychology. Qualitative Research in Psychology, 3(2), 77-101. Retrieved from h t t p : / w w . tandfonline.com/doi / abs/10.1191/1478088706qp063oa

Breakwell, G. M., Hammond, S., Five-Schaw, C., \& Smith, J. A. (2010). Métodos de pesquisa em psicologia (F. R. Elizalde, Trad., 3. ed.). Porto Alegre, RS: Artmed.

Bunge, E., López, P., Mandil, J., Gomar, M., \& Borgialli, R. (2009). Actitudes de los terapeutas argentinos Hacia La incorporación de nuevas tecnologías en psicoterapia. Revista Argentina de Clínica Psicológica, 18(3), 209-216. Retrieved from http://www.redalyc.org/articulo. oa? id=281921775002

Carlino, R. (2011). Distance psychoanalysis: The theory and practice of using communication technology in the clinic (J. Nuss, Trans.) London: Karnac.

Conselho Federal de Psicologia. (2012). Resolução CFP $n^{\circ}$ 011/ 2012. Regulamenta os serviços psicológicos realizados por meios tecnológicos de comunicação a distância, o atendimento psicoterapêutico em caráter experimental e revoga a Resolução CFP N. ${ }^{\circ}$ 12/2005. Brasília, DF: Autor.

Conselho Nacional de Saúde. (2016). Resolução 510 de 07 de abril de 2016. Dispõe sobre as normas aplicáveis a pesquisas em Ciências Humanas e Sociais. Brasília, DF: Presidência da República.

Crestana, T. (2015). Novas abordagens terapêuticas-terapias on-line. Revista Brasileira de Psicoterapia, 17(2), 35-43. Recuperado em http://rbp. celg.org.br/detalhe_artigo.asp?id=176

Distéfano, M. J., Mongelo, M. C., O'Conor, J., \& Lamas, M. C. (2015). Psicoterapia y tecnología: implicancias y desafíos en la inserción de recursos innovadores en la práctica clínica argentina. Revista Electrónica de Psicología Iztacala, 18(4). Retrieved from http://www.revistas. unam.mx/index.php/repi/article/view/53433

Eizirik, C. L., Aguiar, R., \& Schestatsky, S. S. (2005). Psicoterapia de Orientação Analítica: Fundamentos teóricos e clínicos. Porto Alegre, RS: Artmed.

Falcão, L. (2007). Neutralidade e abstinência ontem e hoje. Revista de Psicanálise da SPPA, 14(2), 289-303.

Feijó, L. P., Silva, N. B., \& da Cruz Benetti, S. P. (2018). Experiência e Formação Profissional de Psicoterapeutas Psicanalíticos na Utilização das Tecnologias de Informação e Comunicação. Psicologia: Ciência e Profissão, 38(2), 249-261. http://dx.doi.org/10.1590/1982-3703003032017

Fontanella, B. J. B., Ricas, J., \& Turato, E. R. (2008). Amostragem por saturação em pesquisas qualitativas em saúde: Contribuições teóricas. Cadernos de Saúde Pública, 24(1), 17-27. Recuperado em https://dx.doi.org/10.1590/ S0102-311X2008000100003

Freud. S. (1996a). Recomendações aos jovens médicos que exercem a psicanálise. In Edição Standard Brasileira das Obras Psicológicas Completas de Sigmund Freud. Rio de Janeiro: Imago. (Original publicado em 1912)

Freud, S. (1996b). Recordar, repetir e elaborar. In: In: Edição Standard Brasileira das Obras Psicológicas Completas de Sigmund Freud. Rio de Janeiro, RJ: Imago. (Original publicado em 1914)

Freud, S. (1996c). Linhas de progresso na terapia psicanalítica. In Edição Standard Brasileira das Obras Psicológicas Completas de Sigmund Freud. Rio de Janeiro, RJ: Imago. (Original publicado em 1919)

Freud, S. (1996d). Além do princípio do prazer. In Edição Standard Brasileira das Obras Psicoló- 
gicas Completas de Sigmund Freud. Rio de Janeiro, RJ: Imago. (Original publicado em 1920)

Gabbard, G. O. (2012, November 17). Psychoanalysis in the era of cyberspace. Interview with Glen O. Gabbard/ Interviewer: Sabrina Di Cioccio. In Funzione Gamma: journal on line di psicologia di grupo, Conferência promovida pela APA e D. Winnicott Centre, Departamento of Pediatry and Infant Neuropsychitary de Sapienza. Universidade de Roma. Retrieved from http://www. funzionegamma.it/psychoanalysis-in-the-era-of-cyberspace-interview-with-glen-o-gabbard/

Gabbard, G. O. (2015). Intervenções terapêuticas. O que o terapeuta diz e faz? In G. O. Gabbard, Psicoterapia psicodinâmica de longo prazo. Texto Básico. Porto Alegre, RS: Artmed.

Gabbard, G. O., Kassaw, K. A., \& Perez-Garcia, G. (2011). Professional boundaries in the era of the Internet. Academic Psychiatry, 35(3), 168-174. doi: 10.1176/appi.ap.35.3.168

Garcia, J. (2011). The training of psychoanalysts in Latin American countries without IPA institutions: Antecedents, experiences and problems encountered. International Journal of Psychoanalysis, 92, 715-731. doi: 10.1111/j.17458315.2011.00464.x

Gerhardt, T. E., \& Silveira, D. T. (2009). Métodos de Pesquisa. Porto Alegre, RS: Editora da Universidade Federal do Rio Grande do Sul.

Hallberg, S. C. M. (2015). Percepção e uso de tecnologias da informação e comunicação por psicoterapeutas (Dissertação de mestrado, Pontifícia Universidade Católica do Rio Grande do Sul, Porto Alegre, RS, Brasil).

Hallberg, S. C. M., \& Lisboa, C. S. D. M. (2016). Percepção e uso de tecnologias da informação e comunicação por psicoterapeutas. Temas em Psicologia, 24(4), 1297-1309. doi: https:// dx.doi.org/10.9788/TP2016.4-06

Instituto Brasileiro de Geografia e Estatística. (2013). Pesquisa nacional por amostra de domicílios: Acesso à internet e à televisão e posse de telefone móvel celular para uso pessoal. Recuperado em http://biblioteca.ibge.gov.br/visualizacao/ livros/liv93373.pdf

Kohn, N. V., \& Roth, J. D. (2012). The blank screen of cyberspace. Psychoanalytic Inquiry, 32(5), 426-437. doi: http://dx.doi.org/10.1080/073516 90.2012 .703565
Kowacs, C. (2014). Prática psicanalítica, tecnologia e hipermodernidade. Revista de Psicanálise da SPPA, 21(3), 629. Recuperado em http://revista.sppa.org.br/index.php/RPdaSPPA/article/ view/135/230

Luz, A. B. (2015). Oi. Q horas mesmo ficou nossa sessão? TKS. Revista Brasileira de Psicanálise, 49(1), 165-175.

Machado, D. B., Laskoski, P. B., Severo, C. T., Bassols, A. M., Sfoggia, A., Kowacs, C., ...Eizirik, C. L. (2016). A psychodynamic perspective on a systematic review of Online Psychotherapy for Adults. British Journal of Psychotherapy, 32(1), 79-108. doi: http://onlinelibrary.wiley.com/ doi/10.1111/bjp.12204/abstract

Meissner, W. W. (1998). Neutrality, abstinence, and the therapeutic alliance. Journal of the American Psychoanalytic Association, 46(4), 1089-1128. Retrieved from https://www.ncbi.nlm.nih.gov/ pubmed/9934659

Migone, P. (2013). Psychoanalysis on the Internet: A discussion of its theoretical implications for both online and offline therapeutic technique. Psychoanalytic Psychology, 30(2), 281. doi: http:// dx.doi.org/10.1037/a0031507

Miró, J. (2007). Psicoterapia y nuevas tecnologías. Cuadernos de Medicina Psicosomática y Psiquiatría de Enlace, 81, 15-20. Retrieved from http://in3.uoc.edu/opencms_in3/export/sites/ in3/webs/grups_de_recerca/psinet/_resources/ documents/Miro.pdf

Neves, R. C., \& Silva, M. R. (2014). Reflexões sobre a comunicação por SMS e o processo psicoterápico. Anais do Salão de Iniciação Científica da UFRGS. Porto Alegre, RS. Recuperado em http://www.lume.ufrgs.br/handle/10183/113817

Oliveira, N. H., \& Benetti, S. P. C. (2015). Aliança terapêutica: Estabelecimento, manutenção e rupturas da relação. Arquivos Brasileiros de Psicologia, 67(3), 125-138. Recuperado em http://pepsic.bvsalud.org/scielo.php?script=sci arttext\&pid=S1809-52672015000300010\&lng= pt\&tlng $=\mathrm{pt}$

Pieta, M. A. M., \& Gomes, W. B. (2014). Online therapy: viable or unviable?. Psicologia: Ciência e Profissão, 34(1), 18-31. doi: https://dx.doi. org/10.1590/S1414-98932014000100003

Pieta, M. A. M., Siegmund, G., Gomes, W. B., \& Gauer, G. (2015). Desenvolvimento de protocolos para acompanhamento de psicoterapia 
pela Internet. Contextos Clínicos, 8(2), 128140. Recuperado em http://revistas.unisinos. br/index.php/contextosclinicos/article/view/ ctc. 2015.82 .02

Pires, A. C. J. (2015). Sobre os "tratamentos à distância" em psicoterapia de orientação analítica. Revista Brasileira de Psicoterapia, 17(2), 1121. Recuperado em http://rbp.celg.org.br/detalhe_artigo.asp?id=174

Scharff, J. S. (2013). Psychoanalysis Online: Mental Health, Teletherapy, and Training. London: Karnac.

Sfoggia, A., Kowacs, C., Gastaud, M. B., Laskoski, P. B., Bassols, A. M., Severo, C. T., Machado, D., ...Eizirik, C. L. (2014). Therapeutic relationship on the web: To face or not to face?. Trends in Psychiatry and Psychotherapy, 36(1), 3-10. Retrieved from http://www.scielo.br/ scielo.php? script $=$ sci_arttext\&pid $=$ S223760892014000100003\&lng=en\&tlng=en

Stadter, M. (2013). The influence of social media and the communications technology on self and relationships. In J. S. Scharff (Ed.), Psychoanalysis online: Mental health, teletherapy and training (pp. 3-14). London: Karnac Books.

Sucala, M., Schnur, J. B., Constantino, M. J., Miller, S. J., Brackman, E. H., \& Montgomery, G. H. (2012). The Therapeutic Relationship in E-Therapy for Mental Health: A Systematic Review. Journal of Medical Internet Research, 14(4), e110. Retrieved from http://www. jmir.org/2012/4/e110/
Tong, A., Sainsbury, P., \& Craig, J. (2007). Consolidated criteria for reporting qualitative research (COREQ): A 32-item checklist for interviews and focus groups. International Journal for Quality in Health Care, 19(6), 349-357. doi: 10.1093/intqhe/mzm042

Zamfirescu, V. D. (2013). Freud between the clinical practice and the theory of technique. Romanian Journal of Psychoanalysis/Revue Roumain de Psychanalyse, 6(1).

Zilberstein, K. (2015). Technology, relationships and culture: Clinical and theoretical implications. Clinical Social Work Journal, 43(2), 151158. Retrieved from https://link.springer.com/ article/10.1007/s10615-013-0461-2

Zimerman, D. E. (1999). Fundamentos psicanaliticos: Teoria, técnica, clínica - Uma abordagem didática. Porto Alegre, RS: Artmed.
Recebido: $28 / 07 / 2017$

$1^{a}$ revisão: $17 / 10 / 2017$

Aceite final: 17/10/2017

(cc)BY (c) O(s) autor(es), 2018. Acesso aberto. Este artigo está distribuído nos termos da Licença Internacional Creative Commons Atribuição 4.0 (http://creativecommons.org/licenses/by/4.0/), que permite o uso, distribuição e reprodução sem restrições em qualquer meio, desde que você dê crédito apropriado ao(s) autor(es) original(ais) e à fonte, fornecer um link para a licença Creative Commons e indicar se as alterações foram feitas. 